\title{
PERCEPCÃO dA INFLUÊNCIA DAS POLÍTICAS E PRÁTICAS DE RECURSOS HUMANOS NA SATISFAÇÃO COM O TRABALHO
}

THE INFLUENCE OF PERCEPTION POLICIES AND PRACTICES OF HUMAN RESOURCES IN SATISFACTION WITH WORK

Recebido em 08.11.2016. Aprovado em 06.03.2017

Avaliado pelo sistema double blind review

DOI: http://dx.doi.org/10.12712/rpca.v11i1.843

\author{
Luis Eduardo Brandão Paiva \\ eduardobrandao@alu.ufc.br \\ Universidade Federal do Ceará (UFCE), Fortaleza/CE, BRASIL
}

\section{Tereza Cristina Batista de Lima}

tcblima@uol.com.br

Universidade Federal do Ceará (UFCE), Fortaleza/CE, BRASIL

\section{Thiago Sousa de Oliveira}

thiagooliveira@alu.ufc.br

Universidade Federal do Ceará (UFCE), Fortaleza/CE, BRASIL

\section{Suzete Suzana Rocha Pitombeira}

suzetepitombeira@globo.com

Universidade Federal do Ceará (UFCE), Fortaleza/CE, BRASIL

\section{Resumo}

Esta pesquisa tem como objetivo analisar a influência das políticas e práticas de recursos humanos sobre a satisfação no trabalho. Este tema torna-se relevante devido à identificação da satisfação no trabalho como um fator que causa mudanças nas atitudes e saúde do funcionário, uma vez que podem afetar a sua produtividade. Diante disso, foram delineados os seguintes objetivos específicos: (i) identificar as dimensões com maior e menor relevância entre as políticas e práticas de RH; (ii) identificar quais dimensões apresentam maior e menor relevância na satisfação no trabalho; e (iii) analisar a relação entre satisfação no trabalho e a percepção das políticas e práticas de RH. Em relação aos procedimentos metodológicos, esta pesquisa é de natureza quantitativa, descritiva, por meio de uma survey para a coleta de dados, junto aos funcionários de empresas de grande porte que possuem sede no Estado do Ceará. Os resultados obtidos apontam que os funcionários estão indiferentes em relação a sua satisfação com o trabalho, como também se sentem indiferentes quanto às políticas e práticas de recursos humanos. Já a correlação dos dados indica que existe uma conexão moderada entre o fator remuneração e recompensas e a dimensão satisfação com o salário.

Palavras-chave: Percepção. Políticas e Práticas de Recursos Humanos. Satisfação no Trabalho.

\begin{abstract}
This research aims to analyze the influence of human resources policies and practices on job satisfaction. This issue becomes relevant due to the identification of job satisfaction as a factor that causes changes in the attitudes and health of the employees, since they can affect their productivities. In view of this, the following specific objectives were outlined: (i) identify the dimensions with greater and lesser relevance between HR policies and practices; (ii) identify which dimensions present greater and lesser relevance in job satisfaction; and (iii) analyze the relationship between job satisfaction and the perception of HR policies and practices. In relation to the methodological procedures, this research is of a quantitative nature, descriptive, by means of a survey for the collection of data, together with the employees of large companies that have their headquarters in the State of Ceará. The results show that the employees are indifferent about their satisfaction with work, but they also feel indifferent about human resources policies and practices. The correlation of the data indicates that there is a moderate correlation between the remuneration and rewards factor and the satisfaction dimension with the salary.
\end{abstract}

Keywords: Perception. Policies and Practices of Human Resources. Job Satisfaction. 


\section{Introdução}

A satisfação no trabalho pode ser considerada um elemento que impacta a saúde física e mental, as atitudes e o comportamento profissional e social dos indivíduos. É válido destacar que esta satisfação repercute na vida pessoal, nas relações familiares e na execução de tarefas no trabalho, o que, consequentemente, afeta diretamente as organizações. Estudos sistemáticos sobre o tema foram iniciados a partir da década de 1930, o que atraiu a atenção de pesquisadores das áreas da saúde e da psicologia (DEMO, 2010).

Ferreira e Assmar (2004) ressaltam que as condições relativas ao trabalho são compreendidas como os primeiros aspectos avaliados no estabelecimento da satisfação dos funcionários. $\mathrm{O}$ ambiente externo foi considerado como fator de interferência na relação de satisfação e trabalho. Posteriormente, após estudos, feitos por Locke (1969) e Hackman e Oldham (1975), percebeu-se que tanto o ambiente externo quanto as características individuais influenciam no sentimento do funcionário em relação ao trabalho.

Segundo Newstrom (2008) e Demo (2010), pesquisas sobre a satisfação no trabalho consideram a saúde do indivíduo e o seu desempenho de acordo com as atividades que estes exercem. Dessa forma, quando existe a insatisfação do funcionário, percebe-se, portanto, a incidência de absenteísmo, rotatividade, descompromisso atrasos e desânimos. Logo, a abordagem estratégica de Recursos Humanos (RH) ocasionou imposições de mercado, o que fez gerar expectativas de maior satisfação, produtividade e estabilidade dos empregados.

Os desafios competitivos entre as organizações promoveram um ritmo de crescimento acelerado, em que a tecnologia, o capital humano, a reação rápida a ações do mercado e a contenção de custos são questõeschave para a existência de qualquer organização. Nesse sentido, o RH começou a desenvolver tópicos relacionados ao planejamento, recrutamento e seleção, treinamento e desenvolvimento, avaliação de funcionários, remuneração e benefícios, bem como às questões trabalhistas, de modo a adaptar a organização ao cenário competitivo e globalizado (ROESCH, 2005; SNELL; BOHLANDER, 2010).

A gestão de RH busca o desenvolvimento para obter influência entre as políticas e práticas de $\mathrm{RH}$ sobre os resultados individuais e coletivos das organizações. Existem estudos que procuram mostrar a investigação de antecedentes da percepção de políticas como fator primordial à tomada de decisão dos gestores organizacionais (DEMO, 2010).

Nessa perspectiva, é válido ressaltar que o foco sobre a satisfação no trabalho é crescente no âmbito acadêmico e profissional. Esse tema considera, também, a saúde do trabalhador e os impactos que podem ser gerados para o seu desempenho, por meio de atrasos, absenteísmo, rotatividade de funcionários, entre outros. As políticas e práticas de RH têm influências na satisfação com o trabalho pelo meio da aplicação de suas ferramentas (DEMO, 2010; WAGNER; HOLLENBECK, 2012).

Tomando-se como base as discussões abordadas, surge o seguinte questionamento para esta pesquisa: "Qual a influência das políticas e práticas de RH na satisfação com o trabalho?"

No contexto em que se destacam as políticas e práticas de RH nas empresas, assim como o capital humano, considerado fator primordial para a organização alcançar seus objetivos, pode-se evidenciar quais são os impactos positivos e negativos das políticas e práticas de RH na satisfação dos indivíduos no ambiente de trabalho.

Esta pesquisa tem como objetivo geral analisar a influência das políticas e práticas de RH na satisfação com o trabalho. Foram estabelecidos, portanto, os seguintes objetivos específicos: (i) identificar as dimensões com maior e menor relevância entre as políticas e práticas de $\mathrm{RH}$; (ii) identificar quais dimensões apresentam maior e menor relevância na satisfação no trabalho; e (iii) analisar a relação entre satisfação no trabalho e a percepção das políticas e práticas de $\mathrm{RH}$.

Com o intuito de responderaos objetivos estabelecidos, foram delineadas as seguintes hipóteses: (i) o impacto das políticas e práticas de $\mathrm{RH}$ sobre a satisfação no trabalho ocorre por meio das dimensões envolvimento e remuneração e recompensas; (ii) a satisfação no trabalho tem como dimensão de maior relevância a satisfação com o salário; e (iii) as políticas e práticas de RH contribuem positivamente com a satisfação no trabalho.

Além desta seção introdutória, este estudo segmenta- 
se em mais quatro seções. A segunda seção contempla a revisão da literatura, discutindo conceitos e contextualizações da satisfação no trabalho, políticas e práticas e subsistemas de recursos humanos. Em seguida, são destacados os procedimentos metodológicos. A quarta seção considera a análise e discussão dos resultados. Finalmente, a última seção apresenta as conclusões da pesquisa.

\section{Revisão da Literatura}

\section{Satisfação no trabalho}

O trabalho pode influenciar a vida das pessoas de tal forma que possam prevalecer as decisões sobre a organização e as relações sociais entre os indivíduos. Dessa maneira, a satisfação no trabalho adapta o tempo utilizado com a realização de tarefas no âmbito do trabalho, impactando a saúde e o estado emocional do indivíduo (MARTINEZ; PARAGUAY, 2003).

Tomando-se como base a perspectiva de Weiss (2002), o conceito de satisfação no trabalho refere-se a um construto na forma de julgamento, sendo este positivo ou negativo, de um indivíduo, em relação ao seu trabalho ou alguma situação referente à atividade profissional, considerando, sobretudo, o poder da influência dessa avaliação, em que em um extremo encontra-se a satisfação e, em outro, a insatisfação.

Wagner e Hollenbeck (2012) enfatizam que uma maior satisfação no trabalho está diretamente relacionada com o aumento de desempenho. Desta forma, podese apontar que o aumento da satisfação proporciona um melhor desempenho individual, elevando assim os níveis da organização, e isto faz com que os funcionários fiquem predispostos a falar bem do seu trabalho e a ajudar colegas nas suas tarefas.

Rego (2001) enfatiza que a satisfação no trabalho apresenta uma relação com o sentimento de justiça e respeito a que o trabalhador está submetido na organização. Locke (1969), por sua vez, destaca que a satisfação no trabalho pode gerar consequências para a organização e pode impactar a saúde do trabalhador. Assim, a insatisfação no trabalho seria um estado de emoções que não gera prazer, o que proporciona a negação dos valores do trabalhador.

Em face disso, a satisfação e a insatisfação no trabalho não são acontecimentos distintos, mas momentos opostos de um mesmo fenômeno. Locke (1969) evidencia ainda que a satisfação no trabalho é uma situação que envolve a emoção caracterizada pela alegria (satisfação) ou sofrimento (insatisfação) e pode ser descrita como resultado do conceito que o trabalhador tem sobre o seu trabalho.

Weiss (2002) ressalta que a satisfação no trabalho pode ter uma avaliação positiva ou negativa, enfatizando, sobretudo, que o indivíduo tem a sua própria percepção sobre o seu trabalho ou sobre determinada situação de trabalho, a qual pode estar relacionada à visualização de justiça no respectivo ambiente.

As percepções da influência da satisfação no trabalho, na realização das atividades profissionais, mobilizam as organizações a buscarem alternativas que auxiliem no alinhamento das necessidades do indivíduo, objetivando atingir um nível de satisfação que possibilite vantagem competitiva, considerado um importante meio estratégico para alcançar seus objetivos (DEMO, 2010).

Tomando-se como base a perspectiva de Siqueira (2008), é pertinente mostrar que existem seis aspectos do trabalho que influenciam a satisfação: salário, remuneração, compensação; colegas de trabalho; chefia e supervisão; promoções, crescimento, oportunidade de promoção, avanço; natureza do trabalho; e estabilidade no emprego.

A satisfação no trabalho interfere a satisfação com a vida, por meio da totalização dos sentimentos aferidos no trabalho e da sua transição para a vida fora do trabalho e de atitudes decorrentes desta relação, que podem afetar, especificamente, as relações entre $\mathrm{O}$ funcionário e a sua família. A satisfação é indicada conforme um dos três elementos psicossociais da definição de bem-estar no trabalho com a participação do envolvimento, do trabalho e do comprometimento organizacional afetivo (MARTINEZ; PARAGUAY, 2003; SIQUEIRA, 2008).

$\mathrm{Na}$ perspectiva multidimensional, Puente-Palacios e Borges-Andrade (2005) detectaram, por meio de seus estudos, a hipótese da participação de variáveis que apresentam relação com os indivíduos, resultando na percepção de que a satisfação não é justificada por meio de uma forma, individual ou coletiva, mas pela análise do efeito formado pelas variáveis. À medida em que os níveis de satisfação se alteram, ocorrem impactos relativos às ações do funcionário 
com o seu trabalho. São exemplos elementos como envolvimento, compromisso, ânimo, desempenho, rotatividade, absenteísmo e atrasos, violência e, em casos específicos, ocorrência de furtos (NEWSTROM, 2008).

Os aspectos direcionados à saúde e à satisfação no trabalho são consequências da saúde física e mental e da qualidade de vida. O conceito de qualidade de vida no trabalho tem apresentado inúmeras interpretações, porém há alguns quesitos convergentes que são o bem-estar do trabalhador, sua satisfação com a empresa na forma do seu ambiente de trabalho e na ideia de humanização do trabalho (SANT'ANNA; KILIMNIK, 2011).

A associação entre trabalhadores insatisfeitos e problemas de saúde de ordem mental foi observada como a causa de ansiedade depressiva, o que, consequentemente, afeta a organização em momentos difíceis, comprometendo o desempenho individual e organizacional. Quando existe a ocorrência de incertezas, os conflitos de papéis encontram-se como resultados da insatisfação no trabalho, que está relacionada às experiências negativas que podem, por sua vez, aumentar o desgaste com o trabalho. Os aspectos voltados à satisfação no trabalho atuam, sobretudo, como mecanismo de proteção à saúde do indivíduo (MARTINEZ; PARAGUAY, 2003).

Ferreira e Assmar (2004) evidenciam que a literatura mostra uma investigação a respeito das implicações da satisfação nos elementos que são relacionados, como produtividade, absenteísmo e qualidade de vida. Portanto, existe uma divergência da relação satisfação e do desempenho quanto à natureza, em que relatam a intromissão positiva do contentamento no desempenho, podendo-se indicar que o bom desempenho permite a satisfação.

Siqueira e Padovam (2004) relacionam as dimensões para determinar a ocorrência de avaliações prazerosas, e, com isso, a concepção da satisfação é elevada ao nível multidimensional. Os fatores como suporte, liderança e percepção de justiça têm influência sobre a satisfação no trabalho, atingindo, portanto, características individuais relacionadas à positividade. Siqueira (2008) estabelece o contentamento com o trabalho por meio de cinco dimensões: satisfação com os colegas de trabalho, com o salário, com a chefia, com a natureza do trabalho e com as promoções.
O fator suporte aumenta a satisfação no trabalho conforme o funcionário acredita que o seu chefe o apoia e tenta tornar o trabalho uma atividade mais agradável, melhorando as condições de sua realização. Esta atitude tem o intuito de fortalecer as competências de cada trabalhador ao realizar suas atividades (MELEIRO, 2005).

A existência de um estudo com foco exclusivo na relação entre a satisfação e o cumprimento de atividades no trabalho mostrou a complexidade das atividades desempenhadas como moderadoras dessa conexão, visto as correlações mais profundas sendo alcançadas em ocupações de grande complexidade. A investigação permite enfatizar a importância da busca contínua de modelos que conduzam a relação da satisfação e do desempenho com outras variáveis de adequação e ambientais, que podem, portanto, vir a influenciar estes elementos (JUDGE; BONO; LOCKE, 2001).

Essas dimensões podem ser percebidas por meio dos valores organizacionais que a organização apresenta, tais como tendência à inovação, tipo de gestão, eficiência, atenção com os funcionários e com a relação existente entre si. Esses valores organizacionais proporcionam a identificação dos trabalhadores com o trabalho, o que influencia a satisfação com o trabalho (TAMAYO, 1998).

\section{Políticas, práticas e subsistemas de recursos humanos}

A história da área de RH advém da necessidade de registrar os funcionários, ao relacionar as horas trabalhadas, faltas e atrasos, com o intuito de realizar o seu pagamento ou desconto. Contudo, na metade do século XIV, foram atribuídas funções básicas à administração de pessoal, como o recrutamento e a seleção de pessoas, em que o candidato era "aprovado" e, posteriormente, assinava o "contrato de trabalho" no chamado Livro de Escrita Pessoal (MARRAS, 2011).

Dutra (1990) mostra que os processos de gestão administrativa baseiam-se em dois pilares cruciais: as políticas, na forma de; as diretrizes básicas, que são a base para as decisões e comportamentos; e as práticas, constituídas pelos diversos tipos de procedimentos, métodos e técnicas utilizadas para a execução de decisões que norteiam as ações no contexto da organização e no seu relacionamento com o ambiente. 
Conforme Boxall e Purcell (2011), a gestão de recursos humanos trata de políticas e práticas que estão relacionadas à gestão do trabalho e à condução das pessoas para a realização deste. As políticas e práticas utilizam como mecanismos: recrutamento, seleção, implementação, avaliação, motivação, trainning, desenvolvimento e retenção de talentos. A preocupação da gestão de recursos humanos é contratar e gerenciar pessoas. Nesse sentido, com o aumento dos estudos sobre a gestão de pessoas, ampliou-se ainda mais o entendido sobre os subsistemas de $\mathrm{RH}$, e isto proporciona às organizações ferramentas para estruturação e análise dos funcionários.

\section{Subsistemas da área de recursos humanos}

A administração de RH é considerada a junção de subsistemas que, em conjunto, correspondem a cada atividade da área de gestão de pessoas. O comportamento e as decisões estabelecidas pelo setor de RH são guiados por práticas, políticas e diretrizes básicas que as sustentam. Os subsistemas da área de RH são: recrutamento e seleção, remuneração e/ou cargos e salários, benefícios, treinamento e desenvolvimento, avaliação de desempenho, departamento pessoal, higiene e segurança do trabalho, serviços gerais, contencioso trabalhista, assistência social e relações trabalhistas (HÖPNER, 2008; MARRAS, 2011).

Levando em conta a perspectiva de Demo (2010), as políticas e práticas de $\mathrm{RH}$ mais evidentes em pesquisas são: treinamento; desenvolvimento e educação; condições de trabalho; envolvimento, remuneração e recompensas. É válido enfatizar, ainda, o fato de que a justiça organizacional é um meio de promover o bemestar e o desempenho organizacional, os quais devem ser refletidos nas políticas e práticas de $\mathrm{RH}$.

Serão explorados, a seguir, os subsistemas recrutamento e seleção, remuneração e benefícios, treinamento e desenvolvimento, avaliação de desempenho e saúde e segurança no trabalho, adotados nesta pesquisa.

\section{Recrutamento e seleção}

A rapidez em que ocorrem as mudanças de cenário fortalecem, cada vez mais, a crença de que a competitividade se impõe sobre à eficiência. Avaliando esta perspectiva, um dos grandes desafios dos processos de recrutamento e seleção é encontrar o candidato que possa reunir as competências necessárias, o que detém o maior potencial de desenvolvimento à ampliação da produtividade corporativa (CARVALHO; PASSOS; SARAIVA, 2015). Marras (2011) ressalta, também, como fontes de recrutamento os funcionários da própria empresa, banco de dados interno, indicações, cartazes (internos e externo), entidades (sindicatos), agências de emprego, instituições de ensino, consultorias e outras empresas (fornecedores, clientes).

Aseleçãodepessoaléuma tarefa coma responsabilidade de escolher candidatos, encaminhados pelo recrutador, com o intuito de ocupar as vagas abertas. Nesta fase são comparadas as exigências do cargo com as características dos postulantes à vaga. A seleção pode ser baseada em entrevista e diversos testes de habilidades. Os testes mais utilizados são os práticos, situacionais e psicológicos. No processo de recrutamento e seleção existe a percepção da busca sobre a experiência ou potencial de crescimento. A busca excessiva por experiência, que está baseada no passado, pode gerar uma escolha equivocada, como também o olhar único para o potencial a ser desenvolvido (SNELL; BOHLANDER, 2009).

A importância da descrição de cargos e salários nesta fase pode ser refletida no momento de definição sobre a composição da remuneração e dos benefícios, bem como na avaliação dos cargos, conforme a importância da sua atividade para a organização.

\section{Remuneração e benefícios}

Conforme Ivancevich (2008), a compensação é tratada como todo o tipo de recompensa que o indivíduo recebe em função do desempenho do seu trabalho. Ademais, é definida como uma relação de troca, em que a organização oferece uma recompensa, seja financeira ou não, e o trabalhador oferece serviço e lealdade.

O sistema de remuneração divide-se em: remuneração fixa (relacionada à habilidade, competência, função e benefícios); remuneração de acordo com o desempenho (variável, sofrendo alterações conforme a participação acionária) e outras formas que podem ser criadas em função de um resultado extraordinário (WOOD JR; PICARELLI, 2004).

De acordo com Marras (2011), o benefício é um grupo de programas ou planos apresentados, os quais representam um complemento ao sistema salarial. O seu somatório constitui a remuneração do indivíduo 
e esta, por sua vez, tem por objetivo a satisfação, conforme o atendimento das necessidades básicas para auxiliar a retenção da equipe e o aumento da qualidade de vida. $\mathrm{O}$ atendimento dessas necessidades é definido de forma unilateral, o que é necessário para garantir um nível mínimo de qualidade de vida.

Para aumentar a remuneração, o trabalhador busca atingir e superar metas estabelecidas pela organização. $O$ treinamento e o desenvolvimento permitem evolução das habilidades dos funcionários, a fim de que estes possam alcançar metas e superar as expectativas estabelecidas, com a consequência do aumento dos resultados da organização e do impacto na remuneração da equipe quando são realizados os objetivos.

\section{Treinamento e desenvolvimento}

O treinamento é considerado um processo de entendimento cultural, em um período considerado curto, que objetiva transferir conhecimentos, atitudes ou habilidades direcionadas à execução de atividades e à otimização de trabalho. Assim, pode-se produzir um estado de mudança ocasionada pela experiência (MARRAS, 2011).

O treinamento sistemático suscita em uma maior evolução referente às percepções voltadas para o desenvolvimento. Este progresso associa o treinamento com o aprimoramento das potencialidades dos funcionários, tanto no âmbito profissional, quanto no pessoal (SILVA; LUCIO; BARRETO, 2013). A verificação do ambiente relaciona, contudo, tarefas e capacidades profissionais.

A análise das tarefas consiste na revisão da descrição dos cargos e no seu detalhamento para a identificação das atividades relacionadas a um cargo específico, assim como para as suas competências e habilidades imprescindíveis para o seu desempenho. Nesse contexto, tem como finalidade definir o conteúdo exato do treinamento. A avaliação de competência é composta dos elementos importantes para o sucesso de uma atividade, visto que são perceptíveis quais características devem ser abordadas no interior da organização (SNELL; BOHLANDER, 2009).

A análise pessoal determina quais indivíduos necessitam de treinamento, evitando uso de recursos desnecessários. A auto-eficácia mostra-se um bom meio de prever o sucesso do treinamento, sendo considerada uma etapa que avalia as necessidades dos indivíduos e identifica a qualificação destes para o desempenho do trabalho (IVANCEVICH, 2008; SNELL; BOHLANDER, 2009).

Snell e Bohlander (2009) evidenciam o feedback como um propósito de entrelaçar o conhecimento dos resultados e a motivação, contribuindo, portanto, com o foco no que está sendo realizado, segundo a forma desejada e no que está fora do desejado. A técnica de modificação do comportamento está fundamentada como uma fonte de reforço às atividades realizadas que possam ser repetidas no futuro, indicando às atividades indesejadas e a não atribuição de uma recompensa.

\section{Avaliação de desempenho}

Conforme as modificações que foram se tornando mais frequentes, identificou-se a necessidade de um modelo que oferecesse uma avaliação mais precisa do desempenho dos indivíduos. Diante disso, surgiram técnicas de avaliação de mão única, bilateral e $360^{\circ}$. A avaliação de desempenho foi enquadrada como uma forma de controle, desenvolvimento e melhoria de desempenho (BRANDÃO; GUIMARÃES, 2001).

A avaliação de desempenho tem por objetivo analisar, de forma regular e sistemática, o desempenho do indivíduo conforme alguns objetivos, como desenvolvimento, motivação, recursos humanos e planejamento de contratação, comunicações, cumprimento da legislação e pesquisa em gestão de RH (IVANCEVICH, 2008).

Snell e Bohlander (2009) destacam que o feedback das informações, a identificação de pontos já desenvolvidos, a existência de falhas na identificação e a realização de objetivos influenciam no treinamento organizacional, conforme a necessidade da percepção de pontos relacionados à comunicação, permitindo a obtenção de melhorias, com o intuito de organizar um meio em que ocorram discussões entre as pessoas e em que exista um auxílio aos líderes.

Para a realização de atividades direcionadas ao trabalho, é pertinente que sejam oferecidas condições de segurança no trabalho. Em casos que exista risco à saúde, torna-se necessário o estabelecimento de mecanismos de prevenção e correção de ações que venham a causar prejuízo à saúde dos funcionários. 


\section{Saúde e segurança no trabalho}

A segurança e a saúde no trabalho são essenciais à manutenção dos indivíduos, pois garantem o bemestar físico e emocional, que é gerado por meio de favoráveis condições de trabalho. Marras (2011) define que o setor de saúde e segurança no trabalho responde na atuação de prevenção e correção, elaborando estudos constantes e ações que podem evitar acidentes no trabalho, a fim de preservar, principalmente, a saúde do trabalhador.

Marras (2011) mostra que no Brasil a segurança do trabalho é determinada pela Consolidação das Leis Trabalhistas (CLT), constando no seu artigo 163, que é obrigatória à criação da Comissão Interna de Prevenção de Acidentes (CIPA), conforme o que é definido pela Ministério do Trabalho e que regulamenta, por sua vez, a composição e funcionamento desta comissão. As regras são identificadas nas normas regulamentadoras, totalizadas em 29. Dispõe sobre inspeção prévia, embargo ou interdição, serviço especializado em engenharia de segurança e em medicina no trabalho, a CIPA, a utilização de equipamentos de proteção individual, programas de controle médico de saúde operacional, edificações, programa de prevenção de riscos ambientais, instalações e serviços em eletricidade, transporte, movimentação, armazenagem e manuseio de materiais, dentre outras.

A gestão de pessoas contribui intensamente para o avanço das organizações, visto que oferecem mecanismos para exercer o auxílio para os funcionários, podendo-se alcançar o maior índice de desempenho organizacional e individual, o que fortalece a relação entre as partes envolvidas.

\section{Metodologia}

Esta pesquisa se caracteriza por ser descritiva, de natureza quantitativa, motivo o qual quantifica as informações coletadas. Essa definição sobre a natureza da pesquisa auxilia nos resultados, reduz a deturpação dos dados adquiridos e fornece a confiabilidade e a segurança das inferências (RICHARDSON et al., 2011)

Esta pesquisa tem a intenção de descrever e apontar os eventos para conseguir informações sobre a satisfação no trabalho, as políticas e práticas de $\mathrm{RH}$, bem como quais os fatores dimensionais que apresentam maior relevância na amostra pesquisada (COLLIS; HUSSEY, 2005).

O mercado de trabalho detém inúmeras faces na relação do trabalhador com a organização, pois existem variáveis que interferem neste relacionamento. Este estudo contou com a participação de funcionários de empresas situadas nos setores: cafeeiro, educacional, óptico e agrícola. As empresas foram escolhidas aleatoriamente, por conveniência e acessibilidade dos pesquisadores.

Os questionários foram respondidos presencialmente, em que, por meio de um documento oficial, formalizouse o conteúdo e a veracidade desta pesquisa científica. Dessa forma, foi obtida uma amostra de 62 indivíduos, pertencentes a quatro empresas localizadas no Estado do Ceará, município de Fortaleza. Para esta finalidade, foram utilizadas duas escalas já validadas na literatura empírica e conceitual sobre os temas: (i) Escala de Satisfação no Trabalho, que estabeleceu uma visão multidimensional com itens que abordam o conceito teórico das cinco dimensões (SIQUEIRA, 2008); e (ii) Escala de Políticas e Práticas de RH, a qual apresentou seis dimensões, sendo validada nos Estados Unidos, devido à existência de uma lacuna sobre a literatura e a necessidade de medir as percepções dos trabalhadores sobre as políticas e práticas de gestão de recursos humanos (DEMO; ROZZETT, 2012).

A Escala de Satisfação no Trabalho é dividida em 5 fatores, que são: satisfação com os colegas, com o salário, com a chefia, com a natureza do trabalho e com as promoções. Enquanto a Escala de Políticas e Práticas de Recursos Humanos é composta por seis fatores: recrutamento e seleção, envolvimento, treinamento, desenvolvimento e educação, condições de trabalho, avaliação de desempenho e competências e remuneração e recompensas.

Este instrumento tem a possibilidade de ser utilizado em estudos relacionados à Gestão e Recursos Humanos, podendo-se ressaltar o impacto produzido pelas políticas e práticas de $\mathrm{RH}$, e que, consequentemente, afetam nas atitudes e nos comportamentos dos funcionários, influenciando, contudo, na sua continuidade na organização (DEMO; ROZZETT, 2012).

O questionário, envolvendo as duas escalas, é composto por 69 questões. As primeiras questões são 
voltadas para a identificação do cargo, sexo, estado civil e idade do respondente. A segunda parte avalia a satisfação no trabalho, por meio de 25 questões, estabelecida em uma escala likert de 7 pontos, variando entre (1) "totalmente insatisfeito" e (7) "totalmente satisfeito". Enquanto isso, a terceira parte analisa as políticas e práticas de RH - por meio de 40 questões, com as respostas dispostas em uma escala likert estabelecida por 5 pontos, entre (1) "discordo totalmente da afirmativa" e (5) "concordo totalmente com a afirmativa".

A aplicação do instrumento de pesquisa deu-se no período de 10/04/2015 até 01/06/2015, totalizando desta forma, cinquenta e dois dias. A análise dos dados foi realizada por meio de um programa computacional de tratamento estatístico: Statistical Package for the Social Science (SPSS) (versão 21.0).

Para a análise dos dados, utilizou-se como ferramenta para o cálculo da correlação o coeficiente da correlação linear de Pearson, o qual mensura a intensidade, grau de relação entre duas variáveis e a sua força. Quanto mais próximo do valor 1, maior a correlação, em contrapartida, quanto mais distante, menor a correlação (STEVENSON, 2001).

O coeficiente de correlação linear de Pearson é considerado uma ferramenta estatística utilizada para mensurar a força, a intensidade ou o grau de relação linear entre duas variáveis aleatórias (STEVENSON, 2001). No que diz respeito ao tamanho de amostra ser pequeno, para o valor do coeficiente de correlação linear de Pearson ser expressivo é preciso existir elevada magnitude (próximo de 1). Nessa situação, mesmo com o valor do coeficiente de correlação linear de Pearson apresentando relevância estatística, a amostra pode não ser representativa à população.

A amostra foi analisada, em termos numéricos e percentuais, a partir dos dados sociodemográficos obtidos. Foi realizada a distribuição de frequências dos dados, as médias, os desvios-padrão e os percentis da Escala de Satisfação no trabalho e Escala de Políticas e Práticas de RH. Ademais, utilizou-se o coeficiente de correlação linear de Pearson para relacionar as duas escalas (Satisfação no Trabalho e Políticas e Práticas de Recursos Humanos).

\section{Análise e discussão dos resultados}

\section{Dados sociodemográficos}

A amostra dispõe de 62 indivíduos, sendo 35 referentes à empresa $\mathrm{A}(56,45 \%), 10 \mathrm{da}$ empresa $\mathrm{B}(16,13 \%), 7$ da empresa C $(11,29 \%)$ e 10 da empresa D $(16,13)$, respectivamente nos setores: cafeeiro, óptico, agrícola e educacional. Foram analisados aspectos relacionados à satisfação no trabalho e às políticas e práticas de RH.

Foram coletados, também, dados referentes às variáveis socioeconômicas (idade, gênero, estado civil), em que identificou-se que 38 indivíduos são homens (54,84\%) e 24 são mulheres (45,15\%). A média de idade foi de 28 anos, com a variação entre a idade mínima de 19 anos e a idade máxima de 62 anos.

Considerando o estado civil, tem-se 19 respondentes Casado(a)s/União conjugal (31\%), 39 participantes Solteiro(a)s (63\%), 3 respondentes Separado(a)s/ Divorciado(a)s (5\%) e 1 respondente Viúvo(a) (2\%), podendo-se perceber a predominância de indivíduos solteiros. A amostra referente aos indivíduos Separado(a)s/Divorciado(a)s e Viúvo(a) é pequena, quando comparado com os outros estados civis.

Segundo o nível hierárquico, os indivíduos dividem-se em 20 com cargo no nível Auxiliar (32\%), 19 no cargo no nível de Assistente (31\%), 11 no cargo de Analista (18\%), 6 no cargo de Supervisor (10\%), 4 no cargo de Gerente (6\%) e 2 no cargo de Estagiário (3\%).

Ao relacionar o cargo ocupado e o gênero dos indivíduos, identificou-se a seguinte distribuição: quanto às mulheres, tem-se: 9 no cargo de Auxiliar (15\%), 8 no cargo de Assistente (13\%), 8 no cargo de Analista (13\%), 1 no cargo de Supervisor (2\%) e 2 no cargo de Gerente (3\%). Em relação aos homens, destacam-se 11 no cargo de Auxiliar (18\%), 11 no cargo de Assistente (18\%), 3 no cargo de Analista (3\%), 5 no cargo de Supervisor (8\%), 2 no cargo de Gerente (2\%) e 2 no cargo de Estagiário (3\%).

\section{Dimensões da satisfação no trabalho}

Conforme a validação do questionário por Siqueira (2008), a análise dos resultados considera que quanto maior o valor médio, maior será o grau de satisfação do funcionário com a dimensão de seu trabalho. Ressaltase, também, que os valores entre 1 e 3,9 apontam para a insatisfação; entre 4 e 4,9 mostram condição de indiferença; e entre 5 e 7 indicam satisfação. 
Dessa forma, para caracterizar o grau de satisfação, as dimensões de satisfação com os colegas, chefia e natureza do trabalho representaram indicadores que apontam para o estado de satisfação. As dimensões salário e promoções indicaram um estado de indiferença. Todas as dimensões apresentaram alguma resposta com o maior valor fornecido pela escala. A única dimensão que não obteve resposta com o valor mínimo foi a dimensão satisfação com os colegas. É pertinente enfatizar que nenhuma dimensão aponta à insatisfação no trabalho.

A avaliação da satisfação no trabalho possibilita indicar que os trabalhadores se sentem indiferentes quanto a sua satisfação no trabalho, visto que a média geral das dimensões foi representada por uma média igual a 4,93. Os fatores mais relevantes são a satisfação com a chefia e com os colegas.

Considerando o primeiro objetivo proposto desta pesquisa, identificar as dimensões com maior e menor relevância entre as políticas e práticas de $\mathrm{RH}$, vale-se ressaltar que o valor atribuído a variável $\mathrm{N}$ é referente às respostas sobre cada dimensão da Escala de Satisfação no Trabalho, o que permitem mostrar os seguintes resultados obtidos (TABELA 1).

Tabela 1 . Dimensões da satisfação do trabalho

\begin{tabular}{|c|c|c|c|c|c|c|c|c|c|c|}
\hline \multirow{2}{*}{ Dimensão } & \multicolumn{2}{|c|}{$\mathrm{N}$} & \multirow[t]{2}{*}{ Média } & \multirow[t]{2}{*}{ Mediana } & \multirow[t]{2}{*}{$\begin{array}{c}\text { Modelo } \\
\text { padrão }\end{array}$} & \multirow[t]{2}{*}{ Mínimo } & \multirow[t]{2}{*}{ Máximo } & \multicolumn{3}{|c|}{ Percentis } \\
\hline & Válido & Ausente & & & & & & 25 & 50 & 75 \\
\hline $\begin{array}{c}\text { Satisfação } \\
\text { com os } \\
\text { colegas }\end{array}$ & 310 & 0 & 5,3065 & 5 & 1,05187 & 3 & 7 & 5 & 5 & 6 \\
\hline $\begin{array}{l}\text { Satisfação } \\
\text { com o salário }\end{array}$ & 310 & 0 & 4,4065 & 5 & 1,55252 & 1 & 7 & 3 & 5 & 5 \\
\hline $\begin{array}{l}\text { Satisfação } \\
\text { com a Chefia }\end{array}$ & 310 & 0 & 5,3226 & 5 & 1,29692 & 1 & 7 & 5 & 5 & 6 \\
\hline $\begin{array}{c}\text { Satisfação } \\
\text { com a } \\
\text { Natureza do } \\
\text { trabalho }\end{array}$ & 310 & 0 & 5,0935 & 5 & 1,10937 & 1 & 7 & 5 & 5 & 6 \\
\hline $\begin{array}{l}\text { Satisfação } \\
\text { com as } \\
\text { Promoções }\end{array}$ & 310 & 0 & 4,5484 & 5 & 1,36386 & 1 & 7 & 4 & 5 & 5 \\
\hline
\end{tabular}

Fonte: Pesquisa direta dos autores.

Os resultados obtidos sobre a satisfação com a chefia corroboram a Meleiro (2005), a qual mostra o apoio deste como força que fortalece o comprometimento, satisfação e bem-estar do funcionário com a organização. Essa relação, percebida de forma positiva, eleva a sensação de prazer e compromisso com o trabalho do indivíduo.

A dimensão que apresenta menor nível é a satisfação com o salário, com o resultado apontando para a indiferença. Considera, portanto, o contentamento referente ao trabalho realizado, sua capacidade profissional, o custo de vida e o esforço realizado para o desempenho do trabalho.

A dimensão que apresenta um maior nível de satisfação é a satisfação com a chefia, valendo-se ressaltar o contentamento do funcionário com a organização, a capacidade profissional do seu superior, o interesse pelo trabalho dos subordinados e o entendimento entre eles. Os resultados referentes à satisfação com a chefia podem indicar bom relacionamento do chefe com o seu subordinado, e isto significa uma relação de respeito entre as partes envolvidas, o que é um fator que pode ser modificado conforme o estilo de liderança de cada chefe (MELEIRO, 2005). 
Tomando-se como base a primeira hipótese - o impacto das políticas e práticas de RH sobre a satisfação no trabalho ocorre por meio das dimensões envolvimento e remuneração e recompensas, identifica-se que estes fatores são os que representaram maior influência sobre as dimensões da satisfação no trabalho, o que confirma a aceitação da primeira hipótese desta pesquisa.

\section{Fatores das políticas e práticas de recursos humanos}

Considera-se a escala de políticas e práticas de RH com os fatores: recrutamento e seleção; envolvimento; treinamento, desenvolvimento e educação; condições de trabalho; avaliação de desempenho e competências; e remuneração e competências.

Demo (2008) enfatiza que as políticas mais percebidas são as que apresentam maior adequação e eficácia no alcance dos objetivos organizacionais, e que primam pelo bem-estar dos funcionários, acrescentando, contudo, que este deve ser o primeiro objetivo da organização. Com isso, ocorre o alinhamento das práticas para o melhor desempenho do grupo de trabalho, permitindo atingir os objetivos determinados.

Em relação ao segundo objetivo proposto, identificar quais dimensões apresentam maior e menor relevância na satisfação no trabalho, chega-se aos seguintes resultados, conforme observa-se pela Tabela 2.

Tabela 2 . Fatores das políticas e práticas de recursos humano

\begin{tabular}{|c|c|c|c|c|c|c|c|c|c|c|}
\hline \multirow{2}{*}{ Fator } & \multicolumn{2}{|c|}{$\mathrm{N}$} & \multirow{2}{*}{ Média } & \multirow{2}{*}{ Mediana } & \multirow{2}{*}{$\begin{array}{l}\text { Modelo } \\
\text { padrão }\end{array}$} & \multirow{2}{*}{ Mínimo } & \multirow{2}{*}{ Máximo } & \multicolumn{3}{|c|}{ Percentis } \\
\hline & Válido & Ausente & & & & & & 25 & 50 & 75 \\
\hline Recrutamento e seleção & 372 & 372 & 4,0968 & 4 & 1,09214 & 1 & 5 & 4 & 4 & 5 \\
\hline Envolvimento & 744 & 0 & 4,0309 & 4 & 1,08103 & 1 & 5 & 4 & 4 & 5 \\
\hline $\begin{array}{c}\text { Treinamento, } \\
\text { desenvolvimento e } \\
\text { educação }\end{array}$ & 372 & 372 & 3,9059 & 4 & 1,10586 & 1 & 5 & 3 & 4 & 5 \\
\hline Condições de trabalho & 372 & 372 & 4,0269 & 4 & 1,21061 & 1 & 6 & 4 & 4 & 5 \\
\hline $\begin{array}{l}\text { Avaliação de } \\
\text { desempenho e } \\
\text { competências }\end{array}$ & 310 & 434 & 3,8677 & 4 & 1,19813 & 1 & 5 & 3 & 4 & 5 \\
\hline $\begin{array}{l}\text { Remuneração e } \\
\text { recompensas }\end{array}$ & 310 & 434 & 3,3839 & 4 & 1,46956 & 1 & 5 & 2 & 4 & 5 \\
\hline
\end{tabular}

Fonte: Pesquisa direta dos autores.

Para a interpretação dos resultados obtidos, Demo e Rozzett (2012) explicam que conforme o valor da média aumenta, mais o indivíduo pode confirmar a sua concordância com o conteúdo que avalia em cada fator.

Sendo assim, valores entre 4 e 5 indicam concordância; entre 3 e 3,9 apontam indiferença; e entre 1 e 2,9 sinalizam que o participante não percebe a política de RH, tendo o significado que estão sendo desperdiçados recursos ou que existem problemas na comunicação da empresa com o seu funcionário.

Verifica-se, ainda, que as políticas de recrutamento e seleção, envolvimento e condições de trabalho são percebidas pelos indivíduos. Já as políticas de treinamento, desenvolvimento e educação, avaliação de desempenho e competências e remuneração e recompensas são tratadas com indiferença. O fator que obteve maior valor foi o recrutamento e seleção, indicando concordância com as políticas. O fator que obteve o menor valor foi o de remuneração e recompensas. 
A análise dos resultados aponta que os funcionários se sentem indiferentes quanto às políticas e práticas de $\mathrm{RH}$, observando que a média foi igual a 3,88. Nesse contexto, a política mais percebida é a de recrutamento e seleção, não ocorrendo a insatisfação em nenhum fator analisado.

Em relação à segunda hipótese - a satisfação no trabalho tem como dimensão de maior relevância a satisfação com o salário, vale-se ressaltar que salário apresenta o menor valor representativo, e isto permite rejeitar esta hipótese. Nesse sentido, o fator que apresenta maior relevância é a satisfação com a chefia.

\section{Análise entre as dimensões da satisfação no trabalho e das políticas e práticas de recursos humanos}

As dimensões da satisfação no trabalho: colegas, salário, chefia, natureza do trabalho e promoções, e os fatores das políticas e práticas de RH: recrutamento e seleção, envolvimento, treinamento, desenvolvimento e educação, condições de trabalho, avaliação de desempenho e competências e remuneração e recompensas, podem-se correlacionar da seguinte forma (TABELA 3).

Tabela 3 . Correlação entre fatores e dimensões

\begin{tabular}{|c|c|c|c|c|c|c|}
\hline & & $\begin{array}{c}\text { Satisfação } \\
\text { com os } \\
\text { colegas }\end{array}$ & $\begin{array}{c}\text { Satisfação } \\
\text { com o } \\
\text { salário }\end{array}$ & $\begin{array}{c}\text { Satisfação } \\
\text { com a } \\
\text { chefia }\end{array}$ & $\begin{array}{l}\text { Satisfação } \\
\text { com a } \\
\text { natureza do } \\
\text { trabalho }\end{array}$ & $\begin{array}{c}\text { Satisfação } \\
\text { com a } \\
\text { promoções }\end{array}$ \\
\hline \multirow{3}{*}{ Recrutamento e seleção } & $\begin{array}{c}\text { Correlação de } \\
\text { Pearson }\end{array}$ & 0,087 &, $320^{* *}$ &, $243^{* *}$ &, $285^{* *}$ & $263^{* *}$ \\
\hline & Covariância & 0,094 & 0,505 & 0,32 & 0,322 & 0,365 \\
\hline & $\mathrm{N}$ & 310 & 310 & 310 & 310 & 310 \\
\hline \multirow{3}{*}{ Envolvimento } & $\begin{array}{l}\text { Correlação de } \\
\text { Pearson }\end{array}$ &, $127^{*}$ &, $393^{* *}$ &, $400^{* *}$ &, $449^{* *}$ &, $432^{* *}$ \\
\hline & Covariância & 0,146 & 0,67 & 0,569 & 0,547 & 0,647 \\
\hline & $\mathrm{N}$ & 310 & 310 & 310 & 310 & 310 \\
\hline \multirow{3}{*}{$\begin{array}{c}\text { Treinamento, } \\
\text { desenvolvimento e educação }\end{array}$} & $\begin{array}{c}\text { Correlação de } \\
\text { Pearson }\end{array}$ & 0,089 &, $385^{* *}$ &, $366^{* *}$ &, $444^{* *}$ &, $361^{* *}$ \\
\hline & Covariância & 0,104 & 0,667 & 0,529 & 0,55 & 0,55 \\
\hline & $\mathrm{N}$ & 310 & 310 & 310 & 310 & 310 \\
\hline \multirow{3}{*}{ Condição de trabalho } & $\begin{array}{c}\text { Correlação de } \\
\text { Pearson }\end{array}$ & 0,081 &, $372^{* *}$ &, $262^{* *}$ &, $367^{* *}$ &, $346^{* *}$ \\
\hline & Covariância & 0,108 & 0,73 & 0,429 & 0,515 & 0,596 \\
\hline & $\mathrm{N}$ & 310 & 310 & 310 & 310 & 310 \\
\hline \multirow{3}{*}{$\begin{array}{l}\text { Avaliação desempenho e } \\
\text { competências }\end{array}$} & $\begin{array}{l}\text { Correlação de } \\
\text { Pearson }\end{array}$ & 0,022 &, $462^{* *}$ &, $277^{* *}$ &, $416^{* *}$ &, $328^{* *}$ \\
\hline & Covariância & 0,028 & 0,86 & 0,431 & 0,553 & 0,536 \\
\hline & $\mathrm{N}$ & 310 & 310 & 310 & 310 & 310 \\
\hline \multirow{3}{*}{ Remuneração e recompensas } & $\begin{array}{c}\text { Correlação de } \\
\text { Pearson }\end{array}$ &, $123^{*}$ &, $577^{* *}$ &, $317^{* *}$ &, $391^{* *}$ &, $558^{* *}$ \\
\hline & Covariância & 0,189 & 1,316 & 0,604 & 0,637 & 1,119 \\
\hline & $\mathrm{N}$ & 310 & 310 & 310 & 310 & 310 \\
\hline
\end{tabular}

Fonte: Pesquisa direta dos autores. 
Para a análise dos dados, comparam-se cada fator das políticas e práticas de $\mathrm{RH}$ com cada dimensão da satisfação no trabalho, em que foram obtidos os dados referentes à correlação de Pearson, o sigma entre as duas extremidades, a soma dos quadrados e produtos cruzado, covariância e o número de respostas utilizadas para a comparação.

Nesta averiguação são incluídas duas observações referentes à correlação a respeito da sua significância, em que pode ser significativa no nível 0,01 e 0,05 ; sendo representados na análise da correlação de Pearson, respectivamente, com dois asteriscos e um asterisco, medindo a força da relação entre duas variáveis.

De acordo com a avaliação de Bisquerra, Sarriera e Matínez (2007), as variações do coeficiente de Pearson, entre 0,01 e 0,19 são classificadas como associações muito baixas; de 0,20 a 0,39 , baixas; de 0,40 a 0,59 , moderadas, de 0,60 a 0,79 , altas, de 0,80 a 0,99 , muito altas, e 1 como correlação perfeita.

No tocante ao terceiro objetivo proposto, analisar a relação entre satisfação no trabalho e a percepção das políticas e práticas de RH, verifica-se que a correlação que apresenta maior associação é entre a remuneração e recompensas e a satisfação com o salário, com correlação $=0,577$, considerada, portanto, como uma correlação moderada. A que apresenta menor associação é entre a avaliação de desempenho e a satisfação com os colegas, correlação $=0,022$, a qual representa uma associação muito baixa.

As análises dos dados permitiram indicar que a dimensão da satisfação no trabalho que apresenta maior percepção é a satisfação com a chefia e a de menor representatividade é a satisfação com o salário, enquanto que a percepção de insatisfação no trabalho obteve um resultado geral de indiferença. Desse modo, também identifica-se que na percepção de políticas e práticas de $\mathrm{RH}$, o resultado geral é o de indiferença. A partir da correlação dos dados foram encontradas 8 correlações moderadas, 15 correlações baixas e 6 correlações muito baixas, tendo que vista que a remuneração e recompensas e a satisfação com o salário são a que apresentaram maior correlação. Dado as variáveis correlacionadas, observa-se uma variação positiva, mas sem aproximação com uma correlação perfeita. É importante salientar o fato de não existir variáveis independentes e variáveis dependentes, em que dificilmente é determinado que varia em função de quem.
Portanto, no que tange à terceira hipótese - as políticas e práticas de $\mathrm{RH}$ contribuem positivamente com a satisfação no trabalho, vale-se enfatizar que os trabalhadores compreendem que existe a maior incidência de percepções voltadas à classificação das correlações como baixas e muito baixas, não existindo nenhuma correlação alta, muito alta ou perfeita, e isto sugere a não confirmação desta hipótese. A não confirmação da hipótese indica que é necessário estudos que consolidem os resultados apresentados.

\section{Conclusões}

Esta pesquisa objetivou analisar a influência das políticas e práticas de RH na satisfação com o trabalho. Foram utilizadas a Escala de Satisfação no Trabalho e Escala de Políticas e Práticas de RH para a análise dos objetivos específicos que foram delimitados para esta pesquisa.

Foi considerada uma amostra envolvendo 4 empresas no Estado do Ceará, sendo todas empresas privadas. A maioria dos respondentes foram homens. A média de idade dos participantes foi de 28 anos, com idade mínima de 19 anos e máxima de 62 anos.

Quanto ao estado civil ocorreu uma predominância de Solteiros. A amostra referente aos indivíduos Separado(a)s/Divorciado(a)s e Viúvo(a) são pequenas. Identificou-se: 19 indivíduos Casado(a)s/União conjugal (31\%), 39 Solteiro(a)s (63\%), 3 Separado(a) s/Divorciado(a)s (5\%) e 1 Viúvo(a) ( $2 \%$ ).

A primeira hipótese destacou que o impacto das políticas e práticas de RH sobre a satisfação no trabalho ocorreu por meio das dimensões: envolvimento; e remuneração e recompensas. Após a análise dos dados, observou-se que estes fatores foram os que representaram maior influência sobre as dimensões da satisfação no trabalho, confirmando a primeira hipótese desta pesquisa.

Ações voltadas para a projeção de uma maior qualidade do trabalho e integração entre equipes representam um aspecto valioso para o indivíduo, reduzindo turnover, gastos de demissão e admissão, e possivelmente, elevando os resultados das empresas.

A segunda hipótese abordava que a satisfação no trabalho tem como dimensão de maior relevância a satisfação com o salário. Ao observar os resultados desta pesquisa, verificou-se que a dimensão 
satisfação com o salário apresentou o menor valor representativo, não confirmando essa hipótese. Logo, identificou-se maior relevância numérica na satisfação com a chefia. Esse resultado indica que a crença em que a perspectiva salarial representa maior contentamento não se satisfaz, proporcionando que aspectos relacionados à interação entre indivíduos sejam aspectos mais relevante.

A terceira e última hipótese referiu-se à contribuição positiva das políticas e práticas de $\mathrm{RH}$ com a satisfação no trabalho. Nesse sentido, notou-se que os trabalhadores compreenderam, considerando a correlação dos dados, de forma em que ocorre a maior incidência de percepções voltadas para a classificação das correlações como baixas e muito baixas, não existindo nenhuma correlação alta, muito alta ou perfeita, ocasionando a não confirmação desta hipótese. As correlações obtidas não permitiram inferir que existe uma efetiva colaboração entre políticas e práticas de $\mathrm{RH}$ com a satisfação no trabalho.

A não percepção das políticas e práticas de RH é um reflexo da formação da gestão de pessoas no Brasil. As técnicas introduzidas mostram a mudança constante de implementação de conceitos e sistemas que não se adéquam na realidade das organizações, onde não se realiza um bom processo de atração e retenção de pessoas culminando em uma menor qualidade para solucionar questões de sua responsabilidade (WOOD JR.; T, TONELLI, M.J.; COOKE, B, 2012).

No que tange aos objetivos desta pesquisa, pôde-se apontar como objetivo geral - analisar a interferência das políticas e práticas de $\mathrm{RH}$ na satisfação com o trabalho.

Tomando-se como base o primeiro objetivo específico, foram encontradas maiores relevâncias para os indivíduos nas dimensões satisfação com a chefia, com os colegas e a natureza do trabalho. As dimensões satisfação com as promoções e com o salário apresentaram menor relevância, mas não caracterizaram a insatisfação no trabalho. Destacase que grande parte do público participante consta na condição de subordinado, e que pode existir uma relação mais temerosa em relação ao chefe, não havendo a percepção prática da relação chefesubordinado, sendo somente subordinado-chefe.

O segundo objetivo específico, que foi referente à identificação das dimensões que apresentam maior e menor relevância entre as políticas e práticas de $\mathrm{RH}$, permitiu identificar os fatores que apresentaram maior relevância, que foram o recrutamento e seleção, envolvimento e condição de trabalho. Já os de menor relevância foram treinamento, desenvolvimento $\mathrm{e}$ educação, avaliação de desempenho e competências, e remuneração e recompensas.

O terceiro e último objetivo, que relacionou à análise do nível de satisfação com o trabalho e a percepção das políticas e práticas de $\mathrm{RH}$, possibilitou evidenciar que o nível de satisfação com o trabalho foi identificado como indiferente, isto é, nem satisfeito, nem insatisfeito. A percepção das políticas e práticas de RH também teve como resultado a indiferença.

Esta pesquisa tornou-se pertinente por contribuir para as políticas e práticas de $\mathrm{RH}$, indicando, sobretudo, a percepção dos funcionários com a efetividade de sua aplicação, visto que o seu resultado foi indiferente. É preciso alinhar os aspectos gerenciais da área de RH para que as suas ações se tornem mais efetivas e duradouras, evitando mudanças constantes de métodos e técnicas que atuam sobre a gestão de pessoas. Esses resultados tornam-se relevantes para fomentar a adequação entre ações que possam gerar uma melhoria na saúde dos indivíduos, e com isso atingir a função social da organização.

Mesmo com a introdução de futuras técnicas, tornase imprescindível focar a satisfação com as relações interpessoais visando aumentar o envolvimento dos indivíduos com as tarefas, visto que existe uma grande conexão entre os variados processos de uma organização. O reflexo do envolvimento pode ser percebido conforme a organização estrutura o seu recrutamento, selecionando aqueles que irão contribuir de forma não somente individual, mas com o fortalecimento do processo como um todo

Este estudo encontra-se pautado por algumas limitações referentes à amplitude da pesquisa em outras organizações, a fim de consolidar as perspectivas e implicações. Tratando-se de aspectos relacionados à percepção e aos fatores individuais de cada funcionário, espera-se que esta pesquisa contribua para a realização de pesquisas sobre o tema em outras organizações, pois permitirá uma maior compreensão da percepção de satisfação no trabalho e das políticas e práticas de RH. 


\section{Referências}

BISQUERRA, R.; SARRIERA, J. C.; MATÍNEZ, F.

Introdução à Estatística: enfoque informático com o pacote estatístico SPSS. Porto Alegre: Artmed, 2007.

BOXALL, O; PURCELL, J. Strategy and Human

Resource Management. 3th Ed. United Kingdom: Palgrave Macmillan, 2011.

BRANDÃO, Hugo Pena; GUIMARÃES,

Tomás de Aquino. Gestão de competências e gestão de desempenho: tecnologias distintas ou instrumentos de um mesmo constructo? Revista de Administração de Empresas, v. 41, n.1, p. 8-15, jan./mar. 2001.

CARVALHO, I. M. V.; PASSOS, A. E. V. M.; SARAIVA, S. B. C. Recrutamento e seleção por competências. Rio de Janeiro: FGV, 2008. 132 p

COLLIS, J; HUSSEY, R. Pesquisa em

Administração: Um guia prático para alunos de graduação e pós-graduação. Ed. 2. Porto Alegre: Bookman, 2005.

DEMO, G. Políticas de gestão de pessoas, valores pessoais e justiça organizacional. Revista de Administração Mackenzie, v.11, n.5, p.55-81, 2010.

DEMO, G. Desenvolvimento e validação da escala de percepção de políticas e práticas de gestão de pessoas (EPPGP). Revista de Administração Mackenzie, v. 9, n. 6, p. 77-101, 2008.

DEMO, G.; ROZZETT; K. Human Resource Management Policies and Practices (HRMPP):

Scale validation in the United States. International Journal of Strategic Management, San Angelo, v. 12, n. 3, 2012.

DUTRA, J. S. A Utopia da Mudança das Relações de Poder na Gestão de Recursos Humanos. In: FLEURY, M. T. L. (Org.). In: Cultura e Poder nas Organizações. São Paulo: Atlas, 1990.

FERREIRA, M. C.; ASSMAR, E. M. L. Cultura, satisfação e saúde nas organizações . In:

TAMAYO, A (Orgs).Cultura e Saúde nas

Organizações. São Paulo. Ed. Artmed, 520p, 2004.

HACKMAN, J. R.; OLDHAM, G. R. Development of the job diagnostic survey. Journal of Applied Psychology, v. 60, n. 2, p. 159-170, 1975.
HÖPNER, A. Políticas de recursos humanos e qualidade de vida no trabalho em redes hoteleiras. 2008. Dissertação (mestrado) Universidade Federal do Rio Grande do Sul, Escola de Administração, Programa de Pós-graduação em Administração.

IVANCEVICH, J. M. Gestão de recursos humanos. 10. ed. São Paulo, SP: McGraw Hil, 2008.

JUDGE, T.A.; BONO, J.E.; LOCKE, E.A The job satisfaction - job performance relationship: a qualitative and quantitative review. Psychological Bulletin, v.127, n.1.p. 376-407, 2001.

LOCKE, E. A. What is job satisfaction?

Organizational Behaviour Human Performance, v.4, n.4, p.309-336, 1969.

\section{MARRAS, J. P. Administração de Recursos}

Humanos: do operacional ao estratégico. 14. ed. São Paulo: Saraiva, 2011.

MARTINEZ, M. C; PARAGUAY, A. I. B. B. Satisfação e saúde no trabalho - aspectos conceituais e metodológicos. Cadernos de psicologia social do trabalho, v.6, p.59-78, 2003.

MELEIRO, A. R. Bem-estar no trabalho: os aspectos do suporte do supervisor e estilos de liderança. 2005. Dissertação (Mestrado) Universidade Metodista de São Paulo. Faculdade de Psicologia e Fonoaudiologia, Curso de PósGraduação em Psicologia da Saúde.

\section{NEWSTROM, J. W. Comportamento}

organizacional: o comportamento humano no trabalho. 12.ed. São Paulo, SP: McGraw-Hill, 2008.

PUENTE-PALACIOS, K. E.; BORGES-

ANDRADE, J. E. O efeito da interdependência na satisfação de equipes trabalho: um estudo multinível. Revista de Administração Contemporânea, Curitiba, v. 9, n. 3, jul./set. 2005.

REGO, A. Percepções de justiça: estudos de dimensionalização com professores do ensino superior. Psicologia: Teoria e Pesquisa, v.17, n.2, p.119-131, 2001.

RICHARDSON, R. J. et al. Pesquisa social: métodos e técnicas. 3. ed. rev. e ampl. São Paulo, SP: Atlas, p. 334, 2011. 
ROESCH, S. M. A. Projetos de estágio e de pesquisa em administração: guia para estágios, trabalho de conclusão, dissertações e estudos de caso. 3. ed. São Paulo, SP: Atlas, 2005.

SANT'ANNA, A. S.; KILIMNIK, Z. M. Qualidade de vida no trabalho: abordagens e fundamentos. Rio de Janeiro: Elsevier, 2011.

SIQUEIRA, M. M. M. Antecedentes de Comportamento de Cidadania Organizacional. Análise de um Modelo Pós-Cognitivo. 1995. Tese de Doutorado - Universidade de Brasília, Brasília. Comprometimento organizacional. In: SIQUEIRA, M. M. M. (Org.). Medidas do comportamento organizacional: ferramentas de diagnóstico e de gestão. Porto Alegre: Artmed, 2008.

SIQUEIRA, M. M. M.; PADOVAM, V. A. R. Bases teóricas de bem-estar subjetivo, bem-estar psicológico e bem-estar no trabalho. Psicologia: teoria e pesquisa, v. 24, n. 2, p. 201-209, 2008.

SNELL, S.; BOHLANDER, G. W. Administração de recursos humanos. São Paulo, SP: Cengage Learning, 2009.

STEVENSON, W. J. Estatística aplicada à administração. São Paulo: Harbra, 2001.

TAMAYO, A. Valores Organizacionais: sua relação com satisfação no trabalho, cidadania organizacional e comprometimento afetivo. Revista de

Administração, São Paulo v. 33, n.3, p. 56-63, julho/ setembro 1998.

WAGNER, J. A III; Hollenbeck, J. R.

Comportamento organizacional: criando vantagem competitiva. 3. ed. São Paulo, SP: Saraiva, 2012.

WEISS, H. M. Deconstructing job satisfaction:

Separating evaluations, beliefs and affective

experiences. Human Resource Management

Review, v. 12, n. 2, p. 173-194, 2002.

WOOD JR., T.; PICARELLI FILHO, V.

Remuneração por habilidades e por

competências: preparando a organização para a era das empresas de conhecimento intensivo. São Paulo: Atlas, 1999.

WOOD JR.; T, TONELLI, M.J.; COOKE, B. "Para onde vai a gestão de pessoas?" GV-executivo v11, n. 2, p. 20-24, 2012. 